\title{
DETECTION OF HELICOBACTER PYLORI ANTIGEN IN STOOL BY ENZYME-LINKED IMMUNOSORBENT ASSAY AND COMPARISON WITH CONVENTIONAL METHODS
}

\author{
Rajesh Kumar Rajkumar Selvi', Suraj Holalu Shankeregowda², Yuvaraja3 ${ }^{3}$ Subburaj ${ }^{4}$ \\ ${ }_{1}^{1}$ Post Graduate, Department of Microbiology, Vinayaka Mission's Kirupananda Variyar Medical College, Salem. \\ ${ }^{2}$ Assistant Professor, Department of Anaesthesia, KIMS, Bangalore. \\ ${ }^{3}$ Consultant Gastroenterologist, Vinayaka Mission's Hospital, Salem. \\ ${ }^{4}$ Consultant Surgeon, Vinayaka Mission's Hospital, Salem.
}

\begin{abstract}
Helicobacter pylori (H. pylori) bacteria are 'slow' bacterial pathogens and are associated with gastritis, peptic ulcers, gastric adenocarcinoma and gastric Mucosa-Associated Lymphoid Type (MALT) B-cell lymphomas. Several methods, both invasive and noninvasive, are available for detection of H. pylori infection. Invasive methods involve endoscopy and examination of gastric biopsies, e.g. by culture, rapid urease test or histology and are not appropriate for large-scale population studies. Non-invasive methods include the urea breath test, serology and stool antigen test. The latter approach is non-invasive, does not require highly specialized equipment and unlike serology is more likely to provide evidence of active rather than past infection. Furthermore, it may be more appropriate for use in paediatric patients, where techniques such as serology are insensitive and invasive methods are undesirable. Additionally, it may be used for treatment follow-up purposes. Pathogen-specific stool antigen tests are a valid alternative to the Urea Breath Test for non-invasive detection of H. pylori.
\end{abstract}

\section{METHODOLOGY}

A total of 120 patients who underwent upper gastrointestinal endoscopy for various gastrointestinal disturbances like dyspepsia were included in the study. Stool samples were obtained from the patient on the day of endoscopy and stored at $-20^{\circ} \mathrm{C}$. Three biopsy samples were collected, two from the gastric antrum and one from the corpus. One biopsy sample from the antrum was used for performing Rapid urease test at the Endoscopy room and the other two samples were placed in $10 \%$ formalin and sent to the laboratory for histopathological examination.

\section{RESULTS}

Sensitivity, specificity, positive and negative predictive values of ELISA was $100 \%, 77 \%, 52 \%$ and $100 \%$ respectively.

\section{CONCLUSION}

H. pylori stool antigen (HpSA) is suitable to use particularly in developing countries and for selection of patients for endoscopy. Detection of HpSA shows high sensitivity and specificity and might be useful for non-invasive diagnosis of H. pylori infection in children and adult patients.

\section{KEYWORDS}

HpSA, Non-Invasive Test, Antigen Detection.

HOW TO CITE THIS ARTICLE: Selvi RKR, Shankeregowda SH, Yuvaraja, et al. Detection of helicobacter pylori antigen in stool by enzyme-linked immunosorbent assay and comparison with conventional methods. J. Evolution Med. Dent. Sci. 2016;5(51):33083311, DOI: $10.14260 /$ jemds/2016/766

\section{INTRODUCTION}

Helicobacter pylori (H. pylori) bacteria are 'slow' bacterial pathogens and the name comes from Latin meaning 'spiral rod of the lower part of the stomach.' It was first isolated in 1983 in Australia by Warren and Marshall and was found to be present in patients suffering from type B gastritis. ${ }^{1}$ The $\mathrm{H}$. pylori have now been associated with gastritis, peptic ulcers, gastric adenocarcinoma and gastric Mucosa-Associated Lymphoid Type (MALT) B-cell lymphomas.

The mechanism of tissue injury are not clearly established, and both bacterial and host factors may be determinants of outcome.

Financial or Other, Competing Interest: None.

Submission 09-05-2016, Peer Review 02-06-2016,

Acceptance 09-06-2016, Published 27-06-2016.

Corresponding Author:

Rajesh Kumar Rajkumar Selvi,

5-229, Vidya Jothi School Compound,

Market Road,

Marthandam-629165,

Kanya Kumari District,

Tamilnadu.

E-mail: rajeshrs927@gmail.com

DOI: $10.14260 /$ jemds $/ 2016 / 766$
H. pylori do not appear to invade tissues, except as an incidental finding. Ammonia, produced by urease and by deaminases, may potentiate neutrophil induced mucosal injury. Urease is a chemoattractant and activator of host phagocytic cells. Both the Cag A and Vac A Proteins are important signalling molecules elaborated by $\mathrm{H}$. pylori and the host mounts antibody response to both. ${ }^{2,3}$ Strains from patients with ulcers or stomach cancer more commonly express Cag A compared with controls.

$\mathrm{H}$. pylori bacterium is a small microaerophilic, nonsporing, Gram-negative curved spiral shaped bacterium that is about $3 \mu \mathrm{m}$ long with a diameter of $0.5 \mu \mathrm{m}$ with multiple unipolar-sheathed flagella. Urease production is a consistent finding in Helicobacter species of humans that colonise the stomach, but is uncommon in species found in the intestines. Treatment of $\mathrm{H}$. pylori infection is more effective than antisecretory non-eradicating therapy in preventing recurrent upper gastrointestinal bleeding from peptic ulcer. Consequently, all patients with peptic ulcer bleeding should be tested for $\mathrm{H}$. pylori and eradication therapy should be prescribed to infected patients. ${ }^{4}$ 
Several methods, both invasive and non-invasive, are available for detection of $\mathrm{H}$. pylori infection. Invasive methods involve endoscopy and examination of gastric biopsies, e.g. by culture, Rapid Urease Test (RUT) or histology, and are not appropriate for large-scale population studies. Non-invasive methods include the Urea Breath Test (UBT), serology and stool antigen test. The latter approach is non-invasive, does not require highly specialized equipment and unlike serology is more likely to provide evidence of active rather than past infection. Furthermore, it may be more appropriate for use in paediatric patients where techniques such as serology are insensitive and invasive methods are undesirable. Additionally, it may be used for treatment follow-up purposes. In the case of upper gastrointestinal bleeding, which is a major cause of morbidity, mortality and medical care costs, peptic ulcer is the most frequent source of bleeding in these patients. Treatment of H. pylori infection is more effective than antisecretory non-eradicating therapy in preventing recurrent upper gastrointestinal bleeding from peptic ulcer. Consequently, all patients with peptic ulcer bleeding should be tested for $\mathrm{H}$. pylori and eradication therapy should be prescribed to infected patients. ${ }^{5}$

Pathogen-specific stool antigen tests are a valid alternative to the Urea Breath Test (UBT) for non-invasive detection of $\mathrm{H}$. pylori. Much experience has been gained with Premium Platinum HpSA (Meridian Diagnostics, Cincinnati, Ohio), the first Enzyme Immunoassay (EIA) available for the identification of $\mathrm{H}$. pylori antigens in fecal samples. This test uses polyclonal anti- $\mathrm{H}$. pylori antibodies and has revealed good overall performance in diagnosing $\mathrm{H}$. pylori infection or evaluating the success of eradication therapy. ${ }^{6}$

\section{AIMS AND OBJECTIVES}

The aim of the study is to detect the presence of Helicobacter pylori antigen in stool by ELISA and compare the results with conventional methods like Rapid Urease Test and Histopathological examination.

\section{MATERIALS AND METHODS}

The study was conducted with the approval from the Institutional Ethical Committee of a Tertiary Health Care Centre at Salem, Tamil Nadu. Permission to conduct the study was sought from the respective hospital authorities. Informed consent was obtained from the patients before their enrolment into the study. A total of 120 patients who underwent upper gastrointestinal endoscopy for various gastrointestinal disturbances like dyspepsia were included in the study.

The details of complete history, clinical feature of the patients subjected to endoscopy were obtained. Patients on antibiotics, active gastrointestinal bleeding and those with history of gastric surgery were excluded from the study. Preinvasive procedure preparation for oesophagogastroduodenoscopy was performed as per norms. Stool sample is obtained from the patient on the day of endoscopy. Three biopsy sample were collected, two from the gastric antrum and one from the corpus. One biopsy sample from the antrum was used for performing Rapid urease test and the other two samples were placed in $10 \%$ formalin and sent for histopathological examination. Stool sample was collected in a sterile container on the day of endoscopy and stored at $-20^{\circ} \mathrm{C}$.

\section{Rapid Urease Test}

The test was done using PYLODRY urease kit. The kit was opened and an antral biopsy sample was placed on the urea strip and one drop of distilled water added followed by closing of the kit. Colour change from yellow to pink at room temperature within 30 minutes was taken as positive.

\section{Histopathology}

One specimen from the gastric antrum and one from the corpus were fixed in $10 \%$ formalin, paraffin sections were made and stained with Haematoxylin and Eosin and examined for Helicobacter pylori by an experienced pathologist.

\section{Enzyme-Linked Immunosorbent Assay}

COPROELISA kit was used for the detection of Helicobacter pylori antigen in the stool samples. The test was performed according to the kit manufacturer's instructions. The absorbance is determined at $450 / 620 \mathrm{~nm}$ and the results recorded. Optical Density (OD) value of less than 0.15 is taken as negative and OD value of more than or equal to 0.15 is taken as positive.

\section{RESULTS}

The patient was classified as Gold standard positive when histopathological examination and urease test were both positive and Gold standard negative when both these tests were negative. Out of the total 120 samples, 30 were positive by both ELISA and gold standard tests; 4 were positive by Gold standard but negative by ELISA; 16 were positive by ELISA but negative by gold standard tests; 70 were negative by both ELISA and gold standard tests.

\begin{tabular}{|c|c|c|c|}
\hline $\begin{array}{l}\text { Endoscopy } \\
\text { Findings }\end{array}$ & Total & $\begin{array}{c}\text { ELISA } \\
\text { Positivity }\end{array}$ & Percentage \\
\hline Gastritis & 56 & 32 & 57.14 \\
\hline Oesophagitis & 38 & 16 & 42.1 \\
\hline Lax LES & 22 & 8 & 36.36 \\
\hline $\begin{array}{c}\text { Duodenitis \& } \\
\text { Duodenal ulcer }\end{array}$ & 18 & 10 & 55.55 \\
\hline Gastric ulcer & 10 & 4 & 40 \\
\hline Normal study & 20 & 2 & 10 \\
\hline \multicolumn{4}{|c|}{ Table 1: ELISA Positivity vs Endoscopic Diagnosis } \\
\hline
\end{tabular}

Table 1 shows $57 \%$ of gastritis patients and $56 \%$ of duodenitis patients are positive for HpSAg by Enzyme-Linked Immunosorbent Assay.

\begin{tabular}{|c|c|c|}
\hline Test Name & Positive No (\%) & Negative No. (\%) \\
\hline ELISA & $46(38.3 \%)$ & $74(61.7 \%)$ \\
\hline Rapid Urease Test & $34(28.3 \%)$ & $86(71.7 \%)$ \\
\hline Histopathology & $24(20 \%)$ & $96(80 \%)$ \\
\hline Table 2: Efficacy of Different Laboratory Tests (N= 120) \\
\hline
\end{tabular}

\begin{tabular}{|c|c|c|}
\hline Sl. No. & Statistical Analysis & Percentage (\%) \\
\hline 1. & Sensitivity & 100 \\
\hline 2. & Specificity & 77 \\
\hline 3. & Positive predictive value & 52 \\
\hline 4. & Negative predictive value & 100 \\
\hline 5. & False positivity & 48 \\
\hline 6. & False negativity & 0 \\
\hline \multicolumn{3}{|c|}{ Table 3: Results from ELISA } \\
\hline
\end{tabular}




\section{DISCUSSION}

The present work is based on comparative evaluation of invasive and non-invasive methods of detecting Helicobacter pylori infection. Two biopsy based tests namely rapid urease test and histopathological examination and one stool antigen EIA were analysed. The conclusions from the study give fruitful thought about the relative merits and demerits of the methods.

Among the total 120 patients, 74 (61.67\%) were males and $46(38.33 \%)$ were females. The maximum number of patients in this study was in the age group 50-59 and is in line with the study conducted by D. Nair et al. ${ }^{7}$ The endoscopic examination of the study population revealed that gastritis accounted for $47 \%$, oesophagitis in $32 \%$, lax LES in $18 \%$ and duodenal ulcer in $15 \%$. Among the patients with peptic ulcer disease the predominant symptom was epigastric pain in $80 \%$ of cases, dyspepsia in $67 \%$, vomiting in $48 \%$, loss of weight in $28 \%$, loss of appetite in $18 \%$, haematemesis in $10 \%$ and melena in $4 \%$ of the cases. Epigastric pain was the predominant symptom among patients with acid peptic disease.

Out of 120 samples studied by Rapid urease test, 34 (28.3\%) were positive. The overall positivity of Rapid Urease Test (RUT) correlated well with reports by Sivaprakash et al $(38.7 \%){ }^{8}$, while it was lower than that reported by Maimooma et al $(65.8 \%) .{ }^{9}$ In the present study, $96 \%$ of the cases were positive within the first 20 minutes. This is comparable to $95 \%$ reported by Sengupta et al.10 Marshall et al using an RUT reported that $75 \%$ of the positive tests are detected within 20 minutes, $92 \%$ at 3 hours and $98 \%$ at 24 hours.

Histology has been considered by some to be the gold standard for detection of H. pylori. Unfortunately, histology is an imperfect gold standard as the detection of $\mathrm{H}$. pylori relies upon a number of issues including the site, number and size of gastric biopsies, the method of staining and the level of experience of the examining pathologist. In the present study, Histopathological examination by Haematoxylin and Eosin was positive in $24(20 \%)$ cases.

In the present study, ELISA was positive in $46(38.3 \%)$ cases. The sensitivity, specificity, positive predictive value and negative predictive values were $100 \%, 77 \%, 52 \%$ and $100 \%$ respectively. This correlates with a study by Rani et al 11 in 2000 with a sensitivity of $100 \%$ and specificity of $57.9 \%$.

Syam et al12 evaluated the Helicobacter Pylori Stool Antigen (HpSA) for the detection of $\mathrm{H}$. pylori infection in 63 dyspeptic patients. The sensitivity and specificity of HpSA test were $66.7 \%$ and $78.9 \%$ respectively. They concluded that HpSA stool test may be useful for the primary diagnosis of $\mathrm{H}$. pylori infection in peptic ulcer.

Arikan et al $^{13}$ conducted a prospective study to examine the reliability of the HpSA test. The HpSA test had a sensitivity of $91 \%$ and specificity of $83 \%$. HpSA test proved to be as equally reliable as pathological examination for confirming the existence of H. pylori in humans. Thus, the HpSA test was an useful method for detecting $\mathrm{H}$. pylori in patients for whom endoscopy was not indicated. Fanti et al (1999) in his study to evaluate EIA for HpSA, found that this method has a sensitivity of $98.2 \%$, negative prediction value of $96.4 \%$, specificity of $93.1 \%$ and a positive prediction value of $96.4 \%{ }^{14}$

Vaira et al (1999). ${ }^{15}$, in a multi-centre prospective study, found a sensitivity rate of $94.1 \%$ and a specificity rate of $91.8 \%$ for HpSA testing.
The HpSA test and the Urea Breath Test (UBT) were conducted 4 weeks after eradication treatment. Sensitivity and specificity of HpSA test was $90 \%$ and $95.3 \%$ respectively and that of UBT was $90 \%$ and $98.9 \%$ respectively. Thus, unlike serologic testing that requires several months to achieve significant reduction in antibody titer, the HpSA and UBT with C. 13 can be used to detect prognosis at 4 weeks after the end of the treatment.

In a study conducted in Iran on 54 patients with gastrointestinal problems by Ebrahami et al shows sensitivity and specificity of HpSA before treatment as 78.6\% and $92.3 \%$ respectively. In 2005 a study was carried out on 100 children with dyspeptic symptoms in Tabriz, Iran. They compared three diagnostic methods (histology, serological test and HpSA). HpSA sensitivity and specificity was $54.8 \%$ and $79.4 \%$ respectively. 16

Inelmen et al 17 evaluated the accuracy of HpSA in the diagnosis of $\mathrm{H}$. pylori infection in 85 elderly patients affected by medication. Among 56 patients who were not taking Proton Pump Inhibitors (PPI), the sensitivity and specificity of the Helicobacter pylori stool antigen test were $76 \%$ and $93 \%$ respectively. Among 29 patients who had received pharmacological therapy with PPIs, the sensitivity and specificity of $\mathrm{HpSA}$ tests were $82 \%$ and $83 \%$ respectively. They concluded that HpSA was a useful test in elderly people. The test was easy, simple to perform and non-invasive.

Forne et al (2000) compared HpSA testing with histological methods, UBT and the rapid urease test for the diagnosis of $\mathrm{H}$. pylori infection and to evaluate the use to determine H. pylori status after treatment. Before treatment, the HpSA test has sensitivity and specificity rates of $89.5 \%$ and $77.8 \%$ respectively. The specificity is lower than that of UBT, histological evaluation and rapid urease test. Within 24 hours after treatment, the sensitivity for HpSA is $0 \%$. Within 6 weeks after treatment, the sensitivity is $70.4 \%$ and $81.6 \%$. Six months after treatment, the sensitivity and specificity is further reduced to $50 \%$ and $79.3 \%{ }^{18}$

Thus, they conclude that the HpSA test is beneficial for the primary diagnosis of $\mathrm{H}$. pylori with a similar sensitivity as other standard tests, but with a lower specificity. HpSA testing is not useful for early monitoring to determine the efficacy of treatment. Within 6 weeks and 6 months after treatment for further evaluation of the result of eradication treatment, HpSA testing is not very accurate compared to the UBT.

In this study, we found that HpSA was positive in $57 \%$ of gastritis cases and $56 \%$ of duodenitis cases. These observations suggest that HpSA is a highly reliable diagnostic method for Acid Peptic disease at primary care level where endoscopy facility is not available.

\section{CONCLUSION}

HpSA is suitable to use particularly in developing countries. Detection of $\mathrm{H}$. pylori antigens using enzyme-linked immunosorbent assay shows a high sensitivity and specificity and might be useful for non-invasive diagnosis of $\mathrm{H}$. pylori infection in children and adult patients. HpSA may be useful particularly in selection of the cases requiring endoscopic examination, in monitoring the response to treatment and in epidemiological studies. We recommend using the stool antigen test as a diagnostic test for $\mathrm{H}$. pylori infection. 


\section{REFERENCES}

1. Warren JR, Marshall BJ. Unidentified curved bacilli on gastric epithelium in active chronic gastritis. Lancet 1983;1(8336):1273-5.

2. Cover TL, Cao P, Murthy UK, et al. Serum neutralizing antibody response to the vacuolating cytotoxin of helicobacter pylori. J Clin Invest 1992;90(3):913-8.

3. Aras RA, Fischer W, Perez-Perez GI, et al. Plasticity of repetitive DNA sequences within a bacterial (type 4) secretion system component. J Exp Med 2003;198(9):1349-60.

4. Murray RP, Rosenthal SK, Kobayashi SG, et al. Campylobacter and helicobacter. In: Medical Microbiology Mosby 2002:288-93.

5. Gisbert JP, Trapero M, Calvet X, et al. Evaluation of three different tests for the detection of stool antigens to diagnose helicobacter pylori infection in patients with upper gastrointestinal bleeding. Aliment Pharmacol Ther 2004;19(8):923-9.

6. Gisbert JP, Pajares JM. Diagnosis of helicobacter pylori infection by stool antigen determination: a systematic review. Am J Gastroenterol 2001;96(10):2829-38.

7. Nair D. Immune response to H. pylori in gastroduodenal disorders. Indian Journal of Medical Microbiology 1997; 15:33-5.

8. Sivaprakash R, Rao UA. Indigenous, simple, sensitive and cost effective urease test in the diagnosis of $\mathrm{H}$. pylori for the developing world. Indian J Med Microbiol 1994;12:111-15.

9. Maimooma M, Habibulla CM, Nandan Singh, et al. Evaluation of methods for detection of $\mathrm{H}$. pylori from human antral mucosa. Indian J Med Microbiol 1994;12:39-43.
10. Sengupta S, Saraswathi K, Varaiya A, et al. Helicobacter pylori in duodenal ulcer disease and its eradication. Indian J Med Micro biol 2002;20(3):163-4.

11. Rani AA. Methods of non-invasive detection of helicobacter pylori: helicobacter pylori antigen in stool (HpSA). Abstract Congres National X PGI-PEGI dan Pertemuan Ilmiah National XI PPHI 2001:p 254.

12. Syam AF, Rani AA, Abdullaa M, et al. Accuracy of H. pylori stool antigen for the detection of $\mathrm{H}$. pylori infection in dyspeptic patients. World J Gastroenterol 2005;11(3):386-8.

13. Arikan S, Kocakusak A, Barut G, et al. Helicobacter pylori stool antigen test: results of a prospective study. Surg Today 2004;34(4):318-22.

14. Fanti L, Mezzi G, Cavellero A, et al. A new simple immunoassay for detecting helicobacter pylori infection: antigen in stool specimens. Digestion 1999;60:456-60.

15. Vaira D, Malfertheiner P, Mégraud F, et al. Diagnosis of helicobacter pylori infection with a new non-invasive antigen-based assay. Lancet 1999;354(9172):30-3.

16. Daryani EN, Fatemi SR, Shirazi MH, et al. Role of fecal helicobacter pylori antigen for diagnosis and follow up after treatment of patients affected with this bacteria. J Med Council of IRI 2003;20(4):247-2510.

17. Inelmen EM, Gasparini G, Sergi G, et al. Evaluation of helicobacter pylori with a stool antigen assay in frail, elderly patients. Scand J Gastroenterol 2005;40(7): 794-9.

18. Forne M, Domínguez J, Fernandez-Bañares F, et al. Accuracy of an enzyme immunoassay for the detection of helicobacter pylori in stool specimens in the diagnosis of infection and post treatment check-up. Am J Gastroenterol 2000;95(9):2200-5. 